\title{
Distribution of electric field in medium voltage cable joint geometry
}

\author{
H. A. Illias, Z. H. Lee, A. H. A. Bakar, H. Mokhlis \\ UMPEDAC \\ Electrical Engineering Department \\ Faculty of Engineering, University of Malaya \\ Kuala Lumpur, Malaysia \\ h.illias@um.edu.my
}

\author{
A. Mohd Ariffin \\ College of Engineering \\ Universiti Tenaga Nasional \\ Selangor, Malaysia
}

\begin{abstract}
Cable joint is used to connect different sections of cable because a cable section is limited to a certain length. The design of a cable joint mainly depends on the cable type, the applied voltage and the cores. These factors contribute to the way of how electric field stress is distributed at the cable joint. If there are defects exist within the cable joint insulation material, the electric field at that region is altered. The alteration may cause electrical discharges to occur within the defects if the electric field magnitude is larger than the breakdown strength at the defect sites. Therefore, this paper investigates the electric field distribution in a medium voltage cable joint in the presence of defects. The investigation was done through modelling a medium voltage (MV) cable joint using finite element analysis (FEA) software. Several parameters such as the defect size and location, insulation material dielectric constant and insulation thickness have been studied of their effects on the electric field distribution at the cable joint. The results obtained may be able to help in the designing of cable joint structures which can reduce the electric field stress.
\end{abstract}

Keywords- medium voltage cable joint, electric field, finite element analysis

\section{INTRODUCTION}

Transmission system plays an essential role in delivering electric power to the distribution system from the generation system to serve customers. The transmission system is unique because it is designed to transfer energy from a point to another point at the speed of light since it does not has long term storage capability for electricity.

Generally, the distance between the generation and distribution systems are very far but the length of high voltage cable manufactured is limited. In order to connect the transmission line together, cable joint is required to connect between two high voltage cables. Since transmission lines are longer than common distribution lines, direct connecting cannot be used. There are many types of cable joints with different structures and functions, such as premolded joints, transition joints etc.

However, cable joint is the weakest point in the transmission line because defects exist at the cable joint may lead to a system fault. Defects exist due to the poor installation, manufacturing process or during in-service. Partial discharge is a common reason which causes failure at the cable joint, which leads to a system breakdown $[1,2]$. There are several types of defects at cable joints such as void, insulation layer tips, insulation incision etc.

Failure of the system due to a cable fault can result in costly and time consuming maintenance as the whole component needs to be replaced. Thus, it is very important to avoid such failure in transmission system before breakdown occurs. This can be done through performing a consistent maintenance on the transmission line by analysis, measurement and performance assessment of cable joints. Therefore, by avoiding system breakdown, maintenance cost can be reduced and reliability of the system can be improved.

Since electricity has many applications, power cables come in many shapes, sizes and configurations. Therefore, there are various types of joint used to connect cables. They differ in the design and the capacity. The types of a cable joint depend on $[3,4]$ :

i. Applied voltage - Cable joints are available for low, medium and high voltage power cables. If a cable joint has a lower power capacity than the cable itself, the joint may fail under the stress of the electrical current.

ii. Structure - Cable joints have different basic structures based on how two cables are connected. Some cable joints simply connect two power cables at one point while some accommodate a cable branching or coming into the main line.

iii. Insulation material - Different types of cable joints are necessary for different types of power cables. Cables can have layers of polymeric insulation and need a cable joint that is compatible with the cable insulation.

iv. Cores - Power cables can have a single or multiple insulated cores. For a cable joint to function well, it must have the same number of cores as the cables it is connecting.

\section{FEA MODEL OF MV CABLE JOINT GEOMETRY}

Figure 1 shows a two-dimensional (2D) axial symmetric model geometry of a medium voltage (MV) cable joint that has been developed using FEA software. The model has been used to simulate the electric field distribution in an MV cable joint structure. Table 1 shows the dielectric constant of each component in the cable joint model. The conductor is applied

This project was sponsored by University of Malaya, Malaysia 
with a $20 \mathrm{kV}$ voltage amplitude, the ferrule electrode is assigned as a floating potential, the semiconducting layer is grounded and all internal boundaries are continuity.

\section{SiMULATION RESUlTS}

The results consist of the simulated electric field and electric potential distributions in the FEA model, with and without the presence of defects. The effects of different insulation material dielectric constant, cavity defect location, cavity radius and the insulation thickness on the electric field magnitude within the cavity in the cable joint are also presented in this section.

\section{A. Electric field and electric potential distributions}

The electric potential and electric field distributions in the FEA model of a medium voltage (MV) cable joint are shown in Figures $2 \mathrm{a}$ and $2 \mathrm{~b}$. In Figure $2 \mathrm{a}$, the electric potential is higher on the conductor and electrode parts but decreasing when it is approaching the outer part of cable joint, which is grounded. This results in the electric field distribution to become lower at the conductor and the electrode, which also reduces the electric field in the surrounding insulation material. This is part of a stress control design in a MV cable joint. However, the electric field is higher within the rubber moulding due to its lower dielectric constant than the semiconducting screen and rubber moulding.

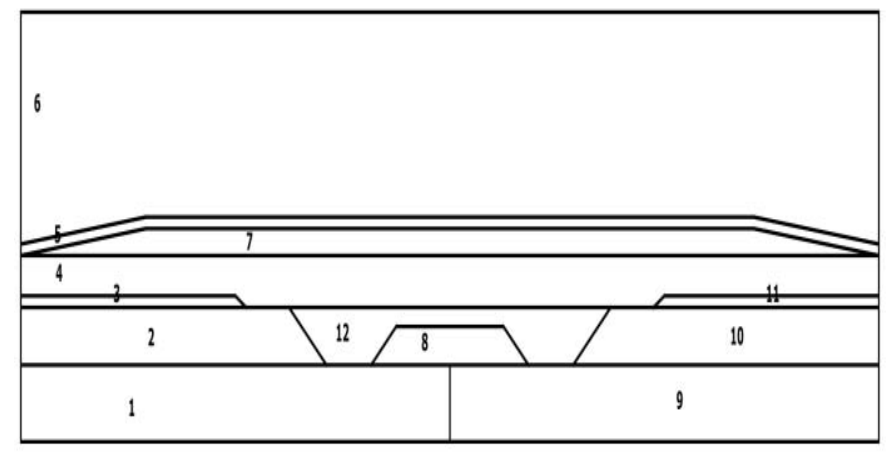

Figure 1. 2D axial-symmetric MV cable joint model geometry using FEA

TABLE I. DiElectric CONSTANT OF COMPONENTS IN MV CABLE JOINT MODEL

\begin{tabular}{|c|c|}
\hline Component & $\begin{array}{c}\text { Dielectric } \\
\text { constant }\end{array}$ \\
\hline Copper conductor (1,9) & 1 \\
\hline XLPE insulation (2,10) & 2.3 \\
\hline Semiconductor screen (3,11) & 2.26 \\
\hline High permittivity layer (4) & 25 \\
\hline Semiconducting layer (5) & 100 \\
\hline Air (6) & 1 \\
\hline Rubber insulation (7) & 1 \\
\hline Ferrule electrode (8) & 50 \\
\hline Ferrule insulation (12) & \\
\hline
\end{tabular}

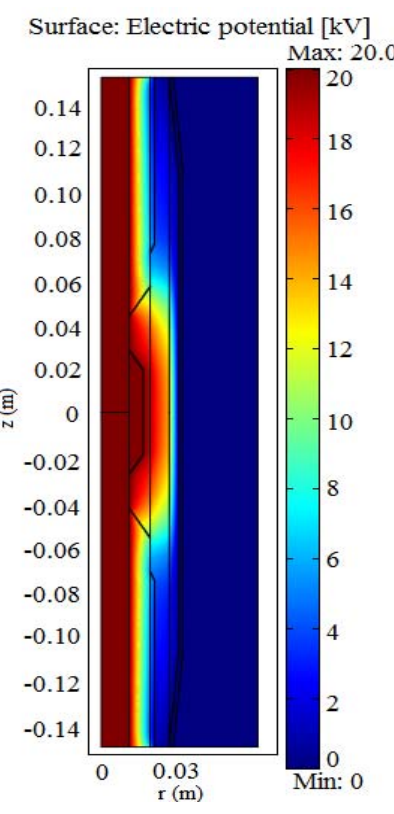

(a)

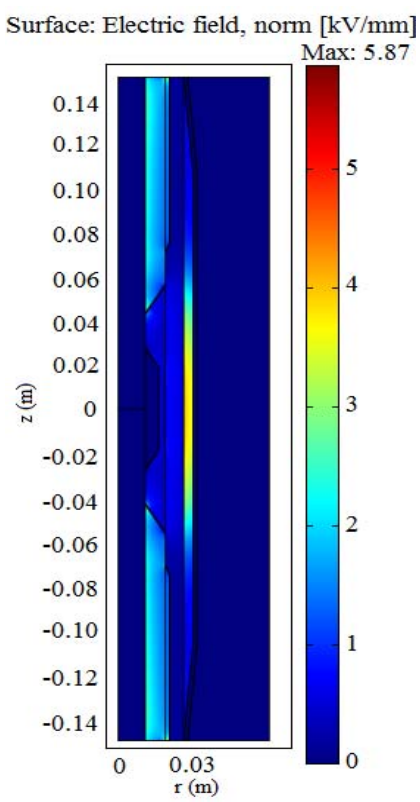

(b)
Figure 2. Simulated (a) electric potential and (b) field distributions

The simulation results of the electric field distribution with various defects in MV cable joint from the FEA model are shown in Figure 3. All defects cause the electric field to become higher at the defect region within the cable joint structure due to the dielectric constant difference between the defect and the material. Figure 4 shows the cross section plot of the electric field magnitude across the MV cable joint between the models with and without different defects. The difference in the electric field magnitude across the cable joint between the presence and the absence of defects can be seen clearly. The discontinuity in the electric field magnitude across the cable joint from $(\mathrm{r}=0.016 \mathrm{~m}$ to $\mathrm{r}=0.030 \mathrm{~m})$ is due to the difference in dielectric constant from different insulation material type. The electric field in the conductor (from $\mathrm{r}=0 \mathrm{~m}$ to $r=0.016 \mathrm{~m}$ ) is zero. In Figure $4 \mathrm{a}$, the cavity region is from $\mathrm{r}$ $=0.021 \mathrm{~m}$ to $\mathrm{r}=0.023 \mathrm{~m}$, in Figure $4 \mathrm{~b}$, the cavity region is from $\mathrm{r}=0.027 \mathrm{~m}$ to $\mathrm{r}=0.029 \mathrm{~m}$, in Figure $4 \mathrm{c}$, the delamination region is from $r=0.026 \mathrm{~m}$ to $\mathrm{r}=0.027 \mathrm{~m}$ and in Figure $4 \mathrm{~d}$, the tip of a sharp pin is at $r=0.031 \mathrm{~m}$.

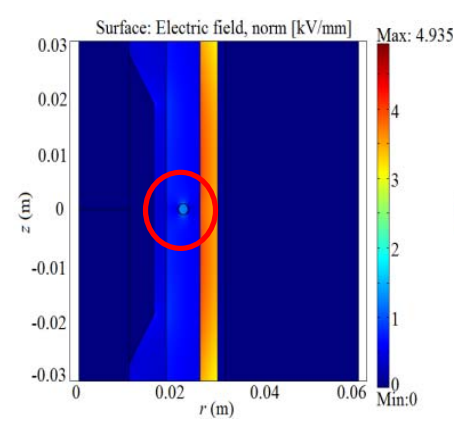

(a) Cavity in high permittivity layer

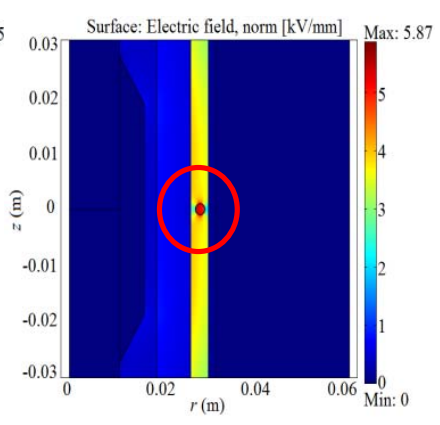

(b) Cavity in rubber insulator 


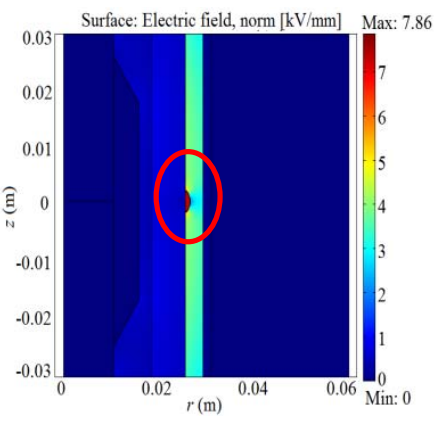

(c) Delamination between two layers

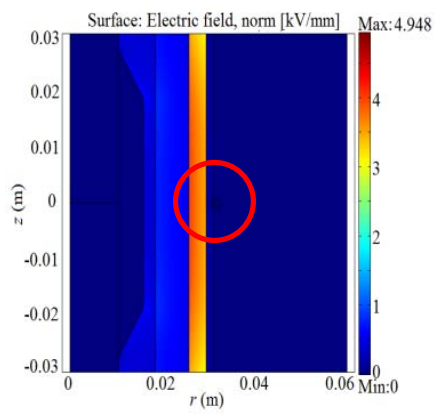

(d) Sharp tip on the surface
Figure 3. Simulated electric field distribution with various defects

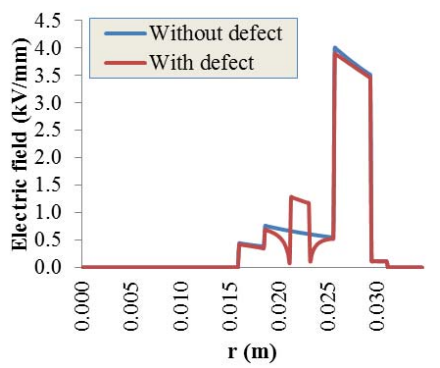

(a) Cavity in high permittivity layer

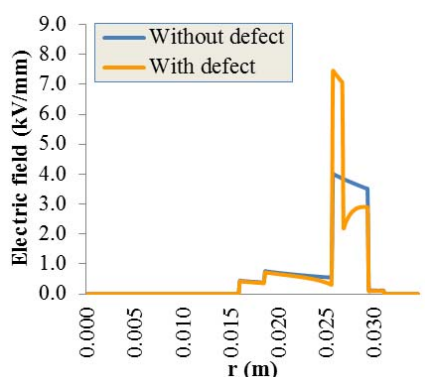

(c) Delamination between two layers

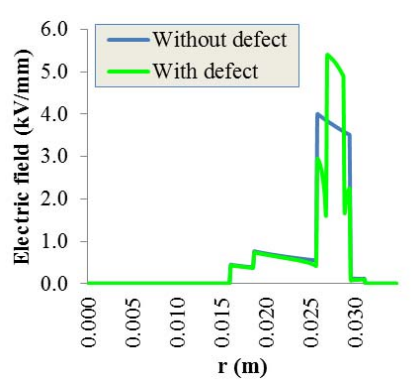

(b) Cavity in rubber insulation

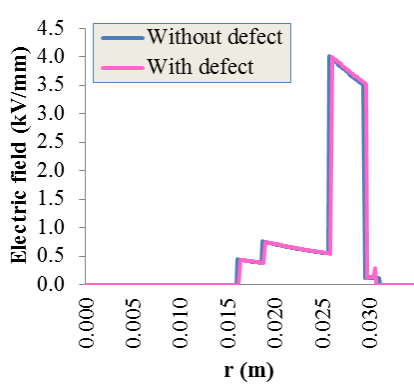

(d) Sharp tip on the surface
Figure 4. Cross section plot of the electric field magnitude in the cable joint models with and without defects along $\mathrm{z}=0$ line

\section{B. Material dielectric constant effect on the electric field}

Figure 5 shows the cross section plots of the electric field magnitude across the medium voltage (MV) cable joint with different values of dielectric constant of different insulation layers. The cross sectional region from $\mathrm{r}=0$ to $0.016 \mathrm{~m}$ consists of the conductor and ferrule electrode, from $r=0.016$ to $0.019 \mathrm{~m}$ consists of the ferrule insulation, from $\mathrm{r}=0.019$ to $0.026 \mathrm{~m}$ consists of the high permittivity layer, from $r=0.026$ to $0.030 \mathrm{~m}$ consists of the rubber moulding and from $\mathrm{r}=0.030$ to $0.031 \mathrm{~m}$ consists of the semiconductor layer. In Figure 5a, it can be seen that the electric field magnitude in the ferrule insulation, rubber moulding and semiconductor layer increases but the electric field in the high permittivity layer decreases as the dielectric constant of the high permittivity layer increases. In Figure 5b, when the dielectric constant of the rubber moulding is increased, the electric field magnitude in ferrule insulation, high permittivity layer and semiconductor layer increases but decreases in the rubber moulding. However, in Figure 5c, the change in the dielectric constant of the semiconducting screen does not affect the electric field magnitude in most of the cable joint parts, except at the semiconducting screen layer.

\section{Effect of cavity locations on the electric field magnitude}

A cavity with a constant radius was introduced in the cable joint FEA models. Its location was varied along the r-axis to study the effect of the cavity location on the electric field within cavity in the cable joint. The plot of the electric field magnitude in the center of the cavity when the cavity location is varied is shown in Figure 6. The magnitude of the electric field decreases when the cavity location is away from central axis of the cable joint. This is because the cavity location is further from the conductor.

The mathematical expression that can represent the electric field magnitude as a function of the cavity location as in Figure 6 is given by

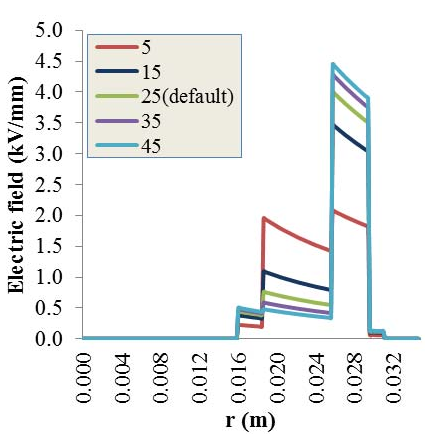

(a) Variation of high permittivity layer dielectric constant

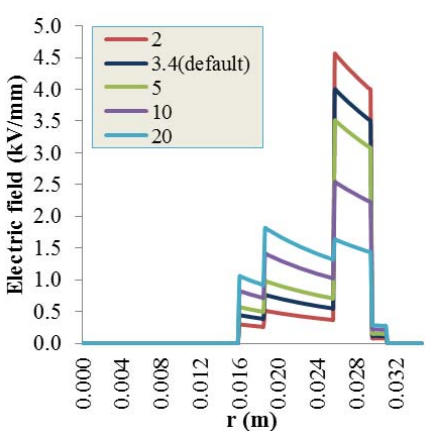

(b) Variation of rubber moulding dielectric constant

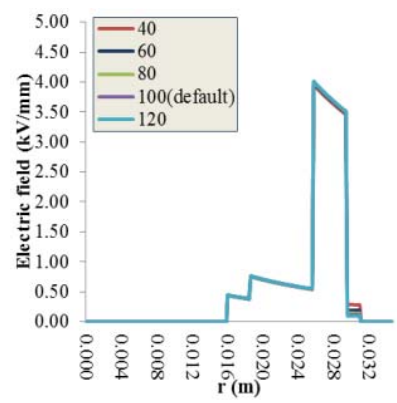

(c) Variation of semiconducting layer dielectric constant

Figure 5. Different material permittivity of different insulation layers in MV cable joint model

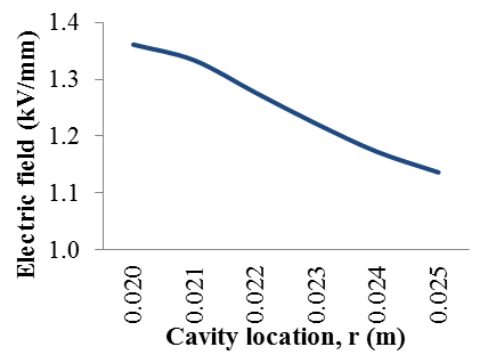

Figure 6. Electric field within the cavity versus cavity location along r-axis 


$$
E=A e^{B r}
$$

where $A$ equals to 2.877 and $B$ equals to $-37.98, E$ is the electric field magnitude in the cavity and $r$ is the cavity location.

\section{Effects of cavity radius on the electric field magnitude}

To study the effect of cavity radius on the electric field magnitude in the centre of the cavity, a cavity with different radius was inserted in the cable joint model at the same location. Figure 7 shows the plot of electric field magnitude against the cavity radius in the FEA cable joint model. From Figure 7, the magnitude of the electric field decreases as the radius of the cavity increases. The electric field equals to $V /(2 r)$, where $V$ is the applied voltage and $2 r$ is the diameter of the cavity. Since the applied voltage is constant, the electric field decreases when the cavity size is larger $[5,6]$.

The best fit mathematical expression for the electric field magnitude as a function of cavity radius can be represented by

$$
\mathrm{E}=A r^{B}+C
$$

where $A$ equals to $-9623, B$ equals to 2.12 and $C$ equals to 1.25 , $E$ is the electric field magnitude in the cavity and $r$ is the radius of the cavity.

\section{E. Effects of insulation thickness on electric field magnitude}

In the study of the effect of the insulation thickness on the electric field within a cavity in cable joint, a constant cavity radius and location in the insulation layer was introduced in the model of different thickness of the insulation layer. Figure 8 shows the plot of the electric field magnitude versus the thickness of the insulation layer in the FEA model of the cable joint. The electric field magnitude decreases when the insulation thickness increases in all models. Since the electric field equals to $V / d$, where $V$ is the applied voltage and $d$ is the material thickness, the magnitude of electric field within the cavity decreases when the insulation thickness increases.

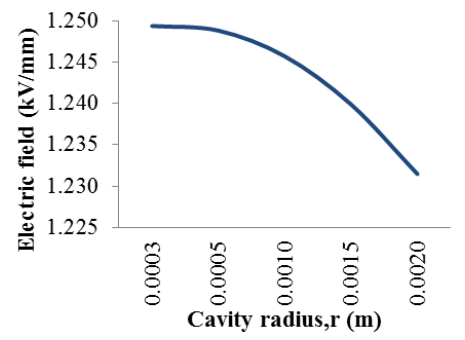

Figure 7. Electric field within the cavity versus cavity radius

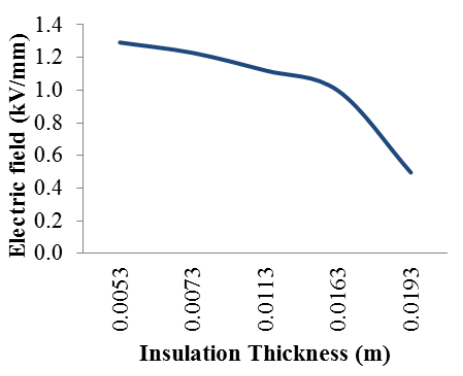

Figure 8. Electric field within the cavity versus insulation thickness

\section{CONCLUSIONS}

From the simulation results that have been obtained, the electric field in the defects in a medium voltage cable joint structure is higher than the surrounding insulation material. This is due to the lower dielectric constant in the defects compared to their surroundings. The effect of different dielectric constant on the electric field magnitude in cable joint depends on its components. The electric field distribution in a cable joint is highly dependent on the dielectric constant of the insulation material. The change in the dielectric constant of the semiconducting layer does not influence the electric field magnitude in other region of the cable joint. However, a higher dielectric constant of the high permittivity layer and rubber moulding causes the electric field magnitude in other regions to become larger but reduces the field within itself.

When the cavity location in the high permittivity layer is further from the central axis of the conductor and the cavity size is larger, the electric field magnitude in the cavity decreases. It was also shown that a thicker insulation material reduces the electric field within a cavity in the insulation. Therefore, this could be one of the ways to reduce the electric field stress in a cable joint design. However, the cost of the cable joint is higher due to a larger volume of the material used.

\section{ACKNOWLEDGMENT}

The author thanks the University of Malaya for supporting this work through the HIR research grant (Grant no: H-1600100-D000048).

\section{REFERENCES}

[1] M. S. Naidu and V. Kamaraju, High Voltage Engineering. 2nd ed., McGraw-Hill, 1996.

[2] E. Kuffel, W. S. Zaengl and J. Kuffel, High Voltage Engineering: Fundamentals. 2nd ed, Butterworth-Heinemann: Newnes, 2000.

[3] W. H. Limited, Cable joints (1.07.01).

[4] UK, T.D., LV-HV Cable Installation, Jointing \& Electrical Distribution Equipment. 2008.

[5] H. Illias, G. Chen and P. L. Lewin, "Modeling of partial discharge activity in spherical cavities within a dielectric material," in IEEE Electrical Insulation Magazine, 27, 2011, pp. 38-45.

[6] H. A. Illias, G. Chen, P. L. Lewin, "Partial discharge within a spherical cavity in a dielectric material as a function of cavity size and material temperature," in IET Science, Measurement and Technology, 6, 2012, pp. 52-62. 